\title{
Dysbiosis of the faecal and biliary microbiotas in biliary tract cancer
}

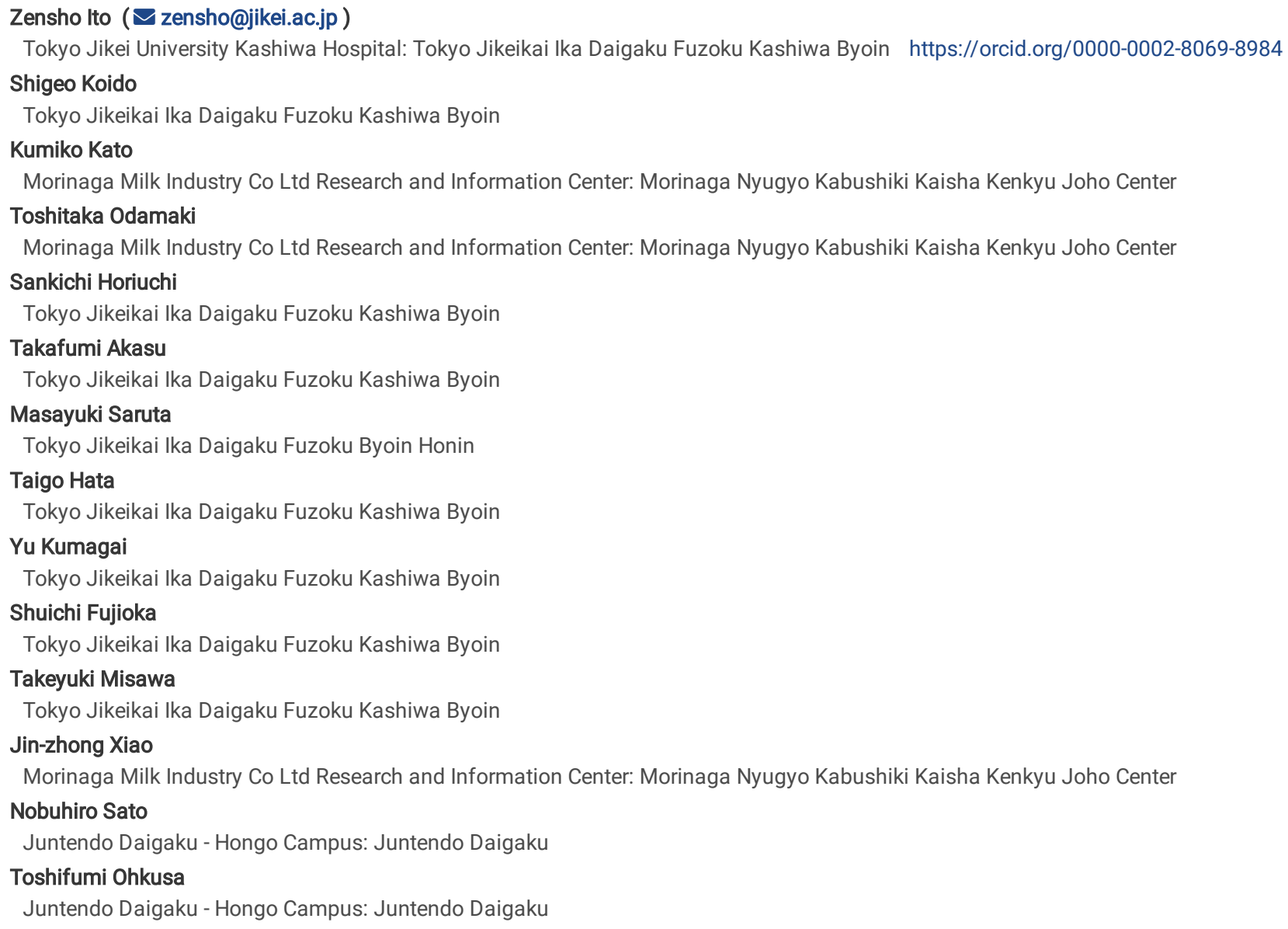

Research

Keywords: BTC, BBD, Linear discriminant analysis (LDA), effect size (LEfSe), operational taxonomic units (OTUs), BRCA1/2, MLH1, MSH2, TP53

Posted Date: June 22nd, 2021

DOI: https://doi.org/10.21203/rs.3.rs-628022/v1

License: @ (1) This work is licensed under a Creative Commons Attribution 4.0 International License. Read Full License 


\section{Abstract \\ Background}

Characteristic bile duct and colonic microbiotas have been identified in patients with chronic biliary tract disease. Therefore, a characteristic microbiota is likely also formed in cholangio- and gallbladder carcinoma patients. This study aimed to characterize the faecal and bile microbiotas in biliary tract cancer (BTC) patients and their relationship to each other.

\section{Methods}

Patients with BTC $(n=30)$ and benign biliary disease (BBD) without cholangitis $(n=11)$ were prospectively included. Ten healthy, age-matched subjects were also recruited for faecal microbiota comparison. The colon and bile duct microbiotas were analysed by sequencing the 16S rRNA gene V3-V4 region. We also obtained live bacteria in the bile collected from three BTC patients by culture and performed metagenomics-based identification.

\section{Results}

The faecal microbiota composition differed in the BTC patients. Linear discriminant analysis (LDA) effect size (LEfSe) showed a higher Enterobacteriaceae abundance and a lower Clostridia abundance, including that of butyrate-producing bacteria including Faecalibacterium and Coprococcus, in the BTC patients than in the other subjects. Notably, 10 of 17 operational taxonomic units (OTUs) assigned to Enterobacteriaceae in the bile samples were matched with the OTUs found in the BTC subject faecal samples. Further metagenomic approaches revealed a bile-isolated strain that possessed the carcinogenic bacterial colipolyketide synthase (PKS)-encoding gene.

\section{Conclusions}

Enterobacteriaceae was enriched in the BTC faeces. Taken together with the finding that more than half of Enterobacteriaceae in the bile matched that in the faeces at the OTU level, our data suggest that faecal microbiota dysbiosis possibly contributes to BTC onset.

\section{Background}

Biliary tract cancer (BTC) is a cancer with a poor prognosis. It is often unresectable at diagnosis and has a 5-year overall survival rate of 10\% or less[1] [2]. The incidence of BTC is considered high, 3 per 100,000 in Hispanic and Asian populations[3] [4], although the incidence is increasing not only in Asia but also in western European countries[2] [5] [6]. BTC has a high potential for metastasis and invasion, and because of its anatomic location and spread along the bile ducts, it is difficult to resect completely by surgery. The standard practice for advanced BTC is cisplatin or gemcitabine, but the response to these chemotherapies is poor, resulting in a 5-year survival rate of just under $10 \%[6]$. Several risk factors for BTC are known. Diseases that increase the risk for BTC include cirrhosis, congenital liver fibrosis, metabolic disease, and liver diseases such as primary sclerosing cholangitis (PSC)[1] [7] [8]. Genetic studies have shown that pathological genetic mutations such as BRCA1/2, MLH1, MSH2, and TP53 are found in BTC[9], but genetic factors alone cannot explain the onset of this disease, and the aetiology of BTC remains unknown.

Recent studies using next-generation sequencing (NGS) have shown that the gut microbiota of patients with liver disease not only is altered at both the upper gastrointestinal and bile duct levels but also promotes changes in the microbiota composition of the colon[10]. In addition, although bile has been widely regarded as sterile, studies in patients with PSC have shown an association between bile acids and bile bacteria[11]. Several bile studies have shown that Proteobacteria, Firmicutes, and Bacteroides are the major constituents in bile[12]. In a study comparing intrahepatic BTC patients and patients with cirrhosis, two genera, Lactobacillus and Alloscardovia, were reported as potential prognostic markers[13]. However, the distribution of the bile microbiota in BTC remains largely unknown. It is therefore essential to identify the components of the bile microbiota and the specific bacteria associated with BTC for prevention and therapeutic development. The purpose of this study was to investigate the faecal and bile microbiotas in selected cohorts of patients experiencing intrahepatic cholangiocarcinoma, extrahepatic cholangiocarcinoma and gallbladder cancer as BTC and cholecystectomy for benign biliary tract disease (BBD). In addition, carcinogenic bacteria in the bile of patients with BTC were studied.

\section{Methods}

Fifty-four consecutive patients were seen for biliary tract diseases. Of these, 13 patients met the following exclusion criteria and were excluded from the analysis. Finally, 30 BTC patients and 11 BBD patients were included. Ten healthy, age-matched subjects were also recruited for faecal microbiota comparison (Figure 1). All patients with biliary tract disease and controls were recruited at Jikei University Kashiwa Hospital. The diagnosis of BTC was based on cholangiography, the presence of typical cholangial lesions on tissue biopsy (if possible), contrast CT findings and elevated tumour markers. The exclusion criteria were patient age less than 18 years, acute bacterial cholangitis, severe medical comorbidities, and previous receipt of treatment interventions, such as endoscopic retrograde cholangiography (ERC) and surgical or anticancer therapy. Patients had to not receive antibiotics within two months prior to participation in the study. This interval was determined to be a sufficient period of time for the gut microbiota to recover from the effects of antibiotic administration. To reduce the effects of geographic conditions, genetic background and diet, all recruited participants were Japanese and had lived in the Kanto region for a number of years. 
Indications for ERC in patients with BTC included the purpose of bile stasis treatment or BTC diagnosis; during ERC, bile aspiration was performed without the use of contrast or interventional antibiotic prophylaxis. No patients with BTC were sampled for acute suppurative cholangitis. The BBD group was established as controls and included those with surgical intent for gallbladder stones or gallbladder polyps. Patients with chronic cholecystitis or acute cholecystitis, a condition that produces persistent inflammation, were excluded. All subjects provided written informed consent, and the study was approved by the clinical research ethics committee of the Jikei University School of Medicine and Kashiwa Hospital, Tokyo Jikei University School of Medicine (number 29-146 (8762)). This study was conducted in accordance with the Declaration of Helsinki.

\section{Analysis of the faecal microbiota}

Faeces were collected on the morning of the hospital visit, and a stool sample aliquot was mixed with 1 ml of guanidine thiocyanate (GuSCN) solution (TechnoSuruga Laboratory Co., Ltd., Shizuoka, Japan), immediately frozen at $-80^{\circ} \mathrm{C}$ and stored until analysis. DNA extraction from the human faecal samples was performed using the bead-beating method as previously described, with some modifications[14]. Briefly, $150 \mu$ l of faecal sample in GuSCN solution was vigorously vortexed with $300 \mathrm{mg}$ of glass beads (AS ONE BZ-01) and $500 \mu$ l of Tris-EDTA (TE, pH 9.0) buffer-saturated phenol (Fujifilm, Wako Pure Chemicals) using a FastPrep-24 (Funakoshi Corporation) for 30 seconds at power level 5. After centrifugation at 10,000×g for $10 \mathrm{~min}, 400 \mu \mathrm{l}$ of the supernatant was extracted with $500 \mu \mathrm{l}$ of phenol-chloroform, and $250 \mu \mathrm{l}$ of the supernatant was precipitated with isopropanol. The purified DNA was suspended in $100 \mu \mathrm{l}$ of TE buffer (pH 8.0).

\section{Bile collection procedure and biological sample acquisition}

Bile was collected during endoscopic or surgical treatment. Bile samples were collected in Techno Suruga Lab containers as in the faecal microbiota analysis. Some bile was also cultured simultaneously with the culture method using the medium described below. All endoscopic surgeries were performed under conscious sedation. Endoscopic retrograde cholangiography was performed with a standard video duodenoscope (TFJ 260-V, Olympus, Tokyo, Japan). A Cook cannula (Cook, Washington, USA) and a Boston guidewire (Boston Scientific, Tokyo, Japan) were used for selective cannulation of the bile duct. A bile sample was aspirated prior to the application of the contrast agent. Antibiotic prophylaxis was applied intravenously after bile samples were obtained and endoscopic scrutiny and treatment were completed. All cholecystectomies or BTC surgeries were performed under general anaesthesia, either open or laparoscopic. Bile samples were aspirated and collected by aseptic manipulation. The biological samples were stored immediately after sampling at $-80^{\circ} \mathrm{C}$ until DNA extraction.

\section{Bile culture assay}

Bile was collected intraoperatively by sterilization from consenting patients with BTC or BBD who underwent surgery. A part of the bile was cultured immediately. For anaerobic culture, bile was collected in Kenky Porter II (KP-C0402, Terumo Co., Ltd.), inoculated with $100 \mu$ l of Kenky Porter II in the following medium and incubated anaerobically for $48 \mathrm{~h}$. The resulting colonies were collected, and DNA was extracted. Sheep blood agar medium (E-MP35, Eiken Chemical), GAM agar medium (05420, Nissui), gentamicin (20 $\mathrm{g} / \mathrm{ml}$ ) (Sigma, G1272)-supplemented GAM agar medium, and FM agar medium (05441, Nissui) were used. For aerobic culture, bile was collected in a stool collection container (Technosulga Lab) for intestinal microbiota testing, inoculated with $100 \mu \mathrm{l}$ of sheep blood agar medium (Eiken Chemical Co., Ltd.) and BTB agar medium (E-MA84, Eiken Chemical Co., Ltd.) and incubated aerobically for $24 \mathrm{~h}$. DNA was extracted from the mixed colonies in each sample.

\section{Microbiota analysis}

Amplicon sequencing of the V3-V4 regions of the bacterial 16S rRNA gene was performed with an Illumina MiSeq instrument, as described previously[14]. Data were analysed in the QIIME2 software package[15] (ver. 2017.10). The reads were mapped to the PhiX 174 sequence and the Genome Reference Consortium human build 38 (GRCh38) by the Bowtie-2 program[16] (ver. 2-2.2.4), and potential chimaeric sequences were removed from acquiring the Illumina paired-end reads by using DADA2[17]. Thereafter, 30 and 90 bases of the 3 ' region of the forward and reverse reads were trimmed, respectively. Taxonomic classification was performed using a naive Bayes classifier trained on Greengenes 13.8[18], with a 99\% threshold for operational taxonomic unit (OTU) full-length sequences. Estimation of alpha diversity and principal coordinate analysis (PCoA) for beta diversity were also performed using QIIME2.

\section{Detection of colipolyketide synthase ( $p k s)$ genomic islands in cultured bacteria isolated from bile acid}

Library construction for an Illumina MiSeq instrument and subsequent de novo assembly of raw reads by the CLC Genomics Workbench ( $v$ 8.0) software package (Qiagen, Valencia, CA) were performed as previously described[19]. Open reading frame (ORF) prediction and annotation were performed using the DDBJ Fast Annotation and Submission Tool (DFAST) with the default settings[20]. Colibactin genomic islands were detected by BLASTP analysis against NCBI reference sequences WP_001217110.1, WP_000357141.1, WP_001518711.1, WP_001297908.1, WP_000982270.1, WP_001297917.1, WP_000337350.1, WP_000159201.1, WP_001304254.1, WP_000829570.1, WP_001468003.1, WP_000222467.1, WP_001297937.1, WP_000217768.1, WP_001327259.1, WP_001029878.1, WP_002430641.1 and WP_000065646.1. PCR for the corroboration of the existence of pks-islands was performed as previously described[21]. The region of primers for the $p k s$-island is shown in Supplementary Figure 1.

\section{Statistical analysis}

Permutational multivariate analysis of variance (PERMANOVA) based on the UniFrac distances was used to evaluate interindividual variability among groups by using the 'adonis' function in the vegan R package (ver. 3.3.0), and p-values of $<0.05$ were considered statistically significant. Linear discriminant analysis (LDA) effect size (LEfSe) was performed with default parameters to identify microbial taxa that were differentially abundant among groups[22].

\section{Data deposition}


DNA sequences corresponding to the 16S rRNA gene and metagenome data have been deposited in DDBJ under accession numbers DRA011518 and DRA011520, respectively.

\section{Results}

\section{Subject background}

Table 1 shows the subject background in addition to blood parameters. There was no significant difference in age $(p=0.073)$, sex $(p=0.079)$ and body-mass index (BMI) ( $p=0.072)$ among the three groups. Some blood parameters were different (Table 1). Cholangitis is a common complication of BTC, and its coexistence is an important concern in the evaluation of the bile microbiota. There was no difference in white blood cell (WBC) levels, as an indicator of the infection status in the BTC group. The decrease in haemoglobin and albumin levels and the increase in C-reactive protein (CRP) levels observed in the BTC group may indicate carcinoma status.

\section{Composition of the faecal microbiota}

We first examined the composition of the faecal microbiota in the BTC, BBD and healthy groups. Both weighted and unweighted UniFrac PCoA showed a significant difference in faecal microbiota composition (Figure 2). LEfSe indicated a higher abundance of Gammaproteobacteria, including Enterobacteriaceae, and a lower abundance of Clostridia, mainly composed of Lachnospiraceae, in the BTC group than in the other groups (Figure 3). Notably, some butyrate-producing bacteria, such as Faecalibacterium and Coprococcus, were enriched in the healthy group. No apparent difference was observed in the alpha diversities based on the Shannon, observed_otus, Chao1 and faith_pd indices (Supplementary Figure 2).

\section{Altered biliary microbiota in patients with BTC}

We then conducted biliary microbiota analysis in eight patients in the BTC group and 10 patients in the BBD group. Of these, we succeeded in the amplification of 16S rRNA genes in four of eight BTC patients and in three of ten BBD patients, indicating bacteria in some of the bile ducts. The bile microbiota varied in the proportion of bacterial species between individuals (Supplementary Figure 3 and Supplementary Table 1), but an OTU assigned to Enterobacteriaceae was enriched in the bile samples of the BTC group compared to the BBD group (Figure 4). To predict the source of the bacteria in the bile duct, we subsequently compared the bile and faecal microbiotas. PCoA showed an obvious difference between them (Supplementary Figure 4); however, there were some common OTUs assigned to Enterobacteriaceae in the faecal and bile microbiotas (Table 2). Notably, the Enterobacteriaceae OTUs were matched in one of eleven (9.1\%) samples in the BBD group and 10 of 19 (52.6\%) samples in the BTC group.

\section{Detection of a pks genomic island in an isolate from bile acid}

To prove the existence of live bacteria in the bile of the patients, we subsequently tried to isolate bacteria from the bile samples. Colonies were detected from the bile samples of subjects 37,40 and 41 . Finally, we performed metagenomics analysis to reveal the features of the isolates from bile acids. Our sequencing effort demonstrated a base sequence-coded pks genomic island, which was responsible for colibactin production, in mixed isolates from the bile acid of subject 41, although the detected pks island was separated on the two contigs (contigs 163 and 33, Supplementary Figure 1). To corroborate the missing area (part of $c / b L$ and $c l b K$ ) in the metagenomic data, we conducted additional PCR using six primer pairs for the whole region of the $p k s$-island, as shown in Supplementary Figure 1. All of the PCR results were positive, suggesting the existence of the whole pks genomic island in the isolate from bile acid. No positive findings were observed in the other bile acid samples.

\section{Discussion}

It has long been suggested that cholecystitis and cholangitis are caused by intestinal bacterial infection. Therefore, we believe that BTC should be investigated in relation to the gastrointestinal microbiota, including the faecal intestinal microbiota and the bile microbiota. In addition, BTC is often accompanied by chronic inflammation of the gallbladder and bile ducts, and chronic inflammation is reported to be caused by infections of intestinal bacteria, including Escherichia coli[1]. In other words, cholecystitis and cholangitis are one of the risk factors for BTC, and intestinal bacteria are the main cause of this risk.

BTC is a disease that occurs in the bile ducts, which have a large mucosal barrier. Diseases that cause chronic inflammation in the bile ducts are known to result in an altered microbiome in the bile ducts. This phenomenon may provide an argument for explaining that cholangitis is one of the risk factors for BTC and may contribute significantly to the nongenetic risk associated with BTC. Previous studies of the microbiome of patients with BTC have reported an increased abundance of Lactobacillus, Actinomyces and others in their gut microbiome[13]. Regarding the bile microbiota, the relative proportion of Fusobacteria, Acidobacteria, Planctomycetes, etc., was reported to be increased in patients with BTC[12]. These reports did not study the bacterial microbiota simultaneously with faeces and bile. Because faeces and bile mutually interact, we felt that it was necessary to study both microbiotas simultaneously in order to study the microbiota constituents responsible for BTC. This is the first study to simultaneously examine the faecal and bile microbiotas in patients with BTC and to more clearly evaluate the microbiotas of BTC patients by performing bile cultures. A variety of factors are known to affect the gut microbiota[23] [24] [25]. Probiotics and antibiotics are also involved in altering the composition and/or metabolites of the gut microbiota[26] [27]. With regard to the history of probiotics and antibiotics, we included individuals who had not received any probiotics or antibiotics in the 2 months prior to sample collection; the 2-month period was established to allow for recovery from the effects of antibiotics on the intestinal microbiota[28]. In this study, the microbiota was analysed among three groups: BTC patients, BBD patients and healthy individuals. Age and sex, which are factors affecting the gut microbiota, were not biased among the three groups. The fact that the background factors were well matched among the three groups allowed us to accurately evaluate the bacterial microbiota in the disease and to analyse it precisely. For the validation of the bile microbiota, we compared the bile microbiota between the BTC and BBD groups. The reason for this exception of healthy subjects was that it is unethical to insert a biliary endoscope into a healthy person for the sole purpose 
of bile collection, which would be highly invasive. The compositions of the faecal microbiota were significantly different (Fig. 2), suggesting that the development of BTC, i.e., harbouring cancer, may lead to changes in the bacterial microbiota at various sites. Therefore, it is worthwhile to examine the faecal microbiota in detail in patients with BTC. The analysis of the cladogram up to the genus level produced by LEfSe for the BTC patients, BBD patients and healthy individuals did not reveal any significant species in the BBD group alone. Enterobacteriaceae was predominantly detected in the BTC group, while Lachnospira, Faecalibacterium, and Coprococcus were significantly enriched in the healthy group (Fig. 3). The bile microbiota was also the focus point in the present study. Surprisingly, we detected the 16S rRNA gene in seven of 18 bile samples from biliary tract disease patients. These results were similar to those reported in Japanese patients with PSC, where bacterial PCR was positive in less than $60 \%$ of cases[29]. The culture method corroborated the existence of live Escherichia coli in three bile samples, although the effect of bile duct organ characteristics on the defence against infection, i.e., bacterial growth in the presence of bile salts, is notable[30]. This result is consistent with the clinical prevalence of Escherichia coli, Klebsiella spp., Enterobacter spp., Enterococcus spp., Streptococcus spp. and other gram-negative bacteria as the causative agents of cholangitis[31]. This could provide an argument for the influence of the gut microbiota on the bile duct microbiota. Similar to the gut-brain axis[32], the gut-bile duct axis might exist. An increased risk of late onset of BTC after papillary sphincteroplasty and common bile duct duodenal anastomosis has been reported, which could provide an argument for the influence of changes in the bile duct microbiota on BTC[33] [34].

Interestingly, 10 of 19 OTUs assigned to Enterobacteriaceae in the bile samples were matched with the OTUs found in the faecal samples of BTC subjects, whereas only one of eleven OTUs was matched in the BBD group. We did not directly prove that any Enterobacteriaceae strain in bile samples had originated from the corresponding faecal samples; however, we believe that the combination findings of the common OTUs assigned to Enterobacteriaceae and live bacteria isolated from the bile samples is a clue to indicate the relationship of Enterobacteriaceae with the onset of BTC.

Finally, we assessed the genetic features of Enterobacteriaceae strains isolated from bile samples in the BTC group. Even though we unfortunately failed to isolate pure strains, our metagenomics data showed the presence of a possible colibactin-producing $E$. coli in a BTC subject. This pathogenic E. coli bacterium produces a hybrid peptide-polyketide genotoxin that causes DNA double-strand breaks and activation of the DNA damage checkpoint pathway, leading to cell cycle arrest. The genetic region responsible for colibactin biosynthesis consists of approximately 20 genes, and its presence has been observed in some $E$. coli strains only[21]. Infection with this colibactin-producing E. coli strain has been shown to increase the degree of carcinogenesis of $E$. coli-associated cancer[35]. To the best of our knowledge, we indicated the presence of $E$. coli possibly carrying the $p k s$ gene island in the bile sample. Whether this bacterium is involved in carcinogenesis in the biliary tract region as well as in colorectal cancer requires further investigation. However, the results of this study will serve as a foundation for the study of the biliary microbiota of the biliary tract.

The limitations of this study are described below. This study was conducted in a single centre, and few cases of the bile microbiota were evaluated from bile culture. However, the novelty of this study is that we were able to analyse the same group of background factors, which allowed us to identify the bacterial microbiota-associated characteristics of BTC. Future studies are needed to determine whether the bacteria carrying the $p k s$ gene are involved in carcinogenesis at the cellular level in BTC. The analysis of the bacterial microbiota of BTC using a larger number of cases from multiple centres is also desirable.

\section{Conclusion}

From this study, we found that the bile microbiota of BTC patients was affected and altered by the faecal microbiota more than that of BBD patients as well as healthy individuals. This result indicates that the gut microbiota, especially the colonic microbiota, may affect the bile microbiota of BTC patients.

\section{Abbreviations}

\section{CA19-9}

carbohydrate antigen $19-9$

BBD

benign biliary disease

BMI

body-mass index

BTC

biliary tract cancer

CEA

carcinoembryonic antigen

CRP

C-reactive protein

ERC

endoscopic retrograde cholangiography

GRCh38

Genome Reference Consortium human build 38

GuSCN

guanidine thiocyanate

LDA

Linear discriminant analysis

LDH 
lactate dehydrogenase

LEfSe

Linear discriminant analysis effect size

ORF

Open reading frame

NGS

next-generation sequencing

OUTs

operational taxonomic units

PCoA

principal coordinate analysis

PERMANOVA

Permutational multivariate analysis of variance

PKS

polyketide synthase

PSC

primary sclerosing cholangitis

WBC

white blood cell

\section{Declarations}

\section{Ethics approval and consent to participate}

This study was reviewed and approved by the ethics committee of the Jikei Institutional Review Board, Jikei University School of Medicine, and by the clinical study committee of Jikei University Kashiwa Hospital [number 29-146(8762)].

\section{Consent for publication}

Not applicable.

\section{Availability of data and material}

No additional data are available.

\section{Competing interests}

The authors have no conflicts of interest to declare.

\section{Funding}

This study was supported in part by the Jikei University Research Fund and Morinaga Milk Co., Ltd.

\section{Authors' contributions}

Zensho Ito, Kumiko Kato and Toshitaka Odamaki analysed the data. Kumiko Kato, Toshitaka Odamaki and Sankichi Horiuchi performed the experiments. Takafumi Akasu, Masayuki Saruta Yu Kumagai, Taigo Hata, Shuichi Fujioka, and Takeyuki Misawa collected the data and discussed the paper. Zensho Ito and Shigeo Koido designed the clinical study. Zensho Ito and Toshitaka Odamaki performed the clinical study and wrote the manuscript. Jin-zhong Xiao, Nobuhiro Sato and Toshifumi Ohkusa critically revised the manuscript for important intellectual content.

\section{Acknowledgements}

We would like to express our deepest appreciation to all the patients and healthy controls for participating in this study.

\section{References}

1. Razumilava N, Gores GJ. Cholangiocarcinoma. Lancet. 2014;383(9935):2168-79. 10.1016/S0140-6736(13)61903-0 [doi].

2. Cunningham SC, Choti MA, Bellavance EC, Pawlik TM. Palliation of hepatic tumors. SurgOncol. 2007;16(4):277-91. S0960-7404(07)00095 - 3 [pii].

3. Tyson GL, El-Serag HB. Risk factors for cholangiocarcinoma. Hepatology. 2011;54(1):173-84. 10.1002/hep.24351 [doi].

4. Everhart JE, Ruhl CE. Burden of digestive diseases in the united states part III: Liver, biliary tract, and pancreas. Gastroenterology. 2009;136(4):1134-44. 10.1053/j.gastro.2009.02.038 [doi].

5. Walter D, Hartmann S, Waidmann O. Update on cholangiocarcinoma: Potential impact of genomic studies on clinical management. ZGastroenterol. 2017;55(6):575-81. 10.1055/s-0043-102581 [doi]. 
6. Doherty B, Nambudiri VE, Palmer WC. Update on the diagnosis and treatment of cholangiocarcinoma. Curr.Gastroenterol.Rep. 2017;19(1):2,017-0542-4. 10.1007/s11894-017-0542-4 [doi].

7. Sekiya S, Suzuki A. Intrahepatic cholangiocarcinoma can arise from notch-mediated conversion of hepatocytes. JClinInvest. 2012;122(11):3914-8. 63065 [pii].

8. Erichsen R, Jepsen P, Vilstrup H, Ekbom A, Sorensen HT. Incidence and prognosis of cholangiocarcinoma in danish patients with and without inflammatory bowel disease: A national cohort study, 1978-2003. EurJEpidemiol. 2009;24(9):513-20. 10.1007/s10654-009-9365-4 [doi].

9. Wardell CP, Fujita M, Yamada T, Simbolo M, Fassan M, Karlic R, et al. Genomic characterization of biliary tract cancers identifies driver genes and predisposing mutations. JHepatol. 2018;68(5):959-69. S0168-8278(18)30021-7 [pii].

10. Kummen M, Holm K, Anmarkrud JA, Nygard S, Vesterhus M, Hoivik ML, et al. The gut microbial profile in patients with primary sclerosing cholangitis is distinct from patients with ulcerative colitis without biliary disease and healthy controls. Gut. 2017;66(4):611-9. 10.1136/gutjnl-2015-310500 [doi].

11. Pereira P, Aho V, Arola J, Boyd S, Jokelainen K, Paulin L, et al. Bile microbiota in primary sclerosing cholangitis: Impact on disease progression and development of biliary dysplasia. PLoS One. 2017;12(8):e0182924. 10.1371/journal.pone.0182924 [doi].

12. Chen B, Fu SW, Lu L, Zhao H. A preliminary study of biliary microbiota in patients with bile duct stones or distal cholangiocarcinoma. BiomedReslnt. 2019;2019:1092563. 10.1155/2019/1092563 [doi].

13. Jia X, Lu S, Zeng Z, Liu Q, Dong Z, Chen Y, et al. Characterization of gut microbiota, bile acid metabolism, and cytokines in intrahepatic cholangiocarcinoma. Hepatology. 2020;71(3):893-906. 10.1002/hep.30852 [doi].

14. Kato K, Ishida S, Tanaka M, Mitsuyama E, Xiao JZ, Odamaki T. Association between functional lactase variants and a high abundance of bifidobacterium in the gut of healthy japanese people. PLoS One. 2018;13(10):e0206189. 10.1371/journal.pone.0206189 [doi].

15. Bolyen E, Rideout JR, Dillon MR, Bokulich NA, Abnet CC, Al-Ghalith GA, et al. Reproducible, interactive, scalable and extensible microbiome data science using QIIME 2. NatBiotechnol. 2019;37(8):852-7. 10.1038/s41587-019-0209-9 [doi].

16. Langmead B, Salzberg SL. Fast gapped-read alignment with bowtie 2. NatMethods. 2012;9(4):357-9. 10.1038/nmeth.1923 [doi].

17. Callahan BJ, McMurdie PJ, Rosen MJ, Han AW, Johnson AJ, Holmes SP. DADA2: High-resolution sample inference from illumina amplicon data. NatMethods. 2016;13(7):581-3. 10.1038/nmeth.3869 [doi].

18. McDonald D, Price MN, Goodrich J, Nawrocki EP, DeSantis TZ, Probst A, et al. An improved greengenes taxonomy with explicit ranks for ecological and evolutionary analyses of bacteria and archaea. ISME J. 2012;6(3):610-8. 10.1038/ismej.2011.139 [doi].

19. Odamaki T, Bottacini F, Kato K, Mitsuyama E, Yoshida K, Horigome A, et al. Genomic diversity and distribution of bifidobacterium longum subsp. longum across the human lifespan. Sci.Rep. 2018;8(1):85,017-18391-x. 10.1038/s41598-017-18391-x [doi].

20. Tanizawa Y, Fujisawa T, Arita M, Nakamura Y. Generating publication-ready prokaryotic genome annotations with DFAST. Methods MolBiol. 2019;1962:215-26. 10.1007/978-1-4939-9173-0\$413 [doi].

21. Nougayrede JP, Homburg S, Taieb F, Boury M, Brzuszkiewicz E, Gottschalk G, et al. Escherichia coli induces DNA double-strand breaks in eukaryotic cells. Science. 2006;313(5788):848-51. 313/5788/848 [pii].

22. Segata N, Izard J, Waldron L, Gevers D, Miropolsky L, Garrett WS, et al. Metagenomic biomarker discovery and explanation. Genome Biol. 2011;12(6):R60,2011-12-6-r60. 10.1186/gb-2011-12-6-r60 [doi].

23. Odamaki T, Kato K, Sugahara H, Hashikura N, Takahashi S, Xiao JZ, et al. Age-related changes in gut microbiota composition from newborn to centenarian: A cross-sectional study. BMC Microbiol 2016;16:90,016-0708-5. 10.1186/s12866-016-0708-5 [doi].

24. Logan AC, Katzman M. Major depressive disorder: Probiotics may be an adjuvant therapy. MedHypotheses. 2005;64(3):533-8. S0306-9877(04)00496-7 [pii].

25. Scott KP, Gratz SW, Sheridan PO, Flint HJ, Duncan SH. The influence of diet on the gut microbiota. PharmacolRes. 2013;69(1):52-60. 10.1016/j.phrs.2012.10.020 [doi].

26. Sugahara H, Odamaki T, Fukuda S, Kato T, Xiao JZ, Abe F, et al. Probiotic bifidobacterium longum alters gut luminal metabolism through modification of the gut microbial community. SciRep. 2015;5:13548. 10.1038/srep13548 [doi].

27. Langdon A, Crook N, Dantas G. The effects of antibiotics on the microbiome throughout development and alternative approaches for therapeutic modulation. Genome Med. 2016;8(1):39,016-0294-z. 10.1186/s13073-016-0294-z [doi].

28. Coker OO, Dai Z, Nie Y, Zhao G, Cao L, Nakatsu G, et al. Mucosal microbiome dysbiosis in gastric carcinogenesis. Gut. 2018;67(6):1024-32. 10.1136/gutjnl-2017-314281 [doi]

29. Hiramatsu K, Harada K, Tsuneyama K, Sasaki M, Fujita S, Hashimoto T, et al. Amplification and sequence analysis of partial bacterial 16S ribosomal RNA gene in gallbladder bile from patients with primary biliary cirrhosis. JHepatol. 2000;33(1):9-18. S0168-8278(00)80153-1 [pii].

30. Sung JY, Costerton JW, Shaffer EA. Defense system in the biliary tract against bacterial infection. DigDisSci. 1992;37(5):689-96. 10.1007/BF01296423 [doi].

31. Arthur JC, Perez-Chanona E, Muhlbauer M, Tomkovich S, Uronis JM, Fan TJ, et al. Intestinal inflammation targets cancer-inducing activity of the microbiota. Science. 2012;338(6103):120-3. 10.1126/science.1224820 [doi].

32. Sagami Y, Shimada Y, Tayama J, Nomura T, Satake M, Endo Y, et al. Effect of a corticotropin releasing hormone receptor antagonist on colonic sensory and motor function in patients with irritable bowel syndrome. Gut. 2004;53(7):958-64. 10.1136/gut.2003.018911 [doi].

33. Hakamada K, Sasaki M, Endoh M, Itoh T, Morita T, Konn M. Late development of bile duct cancer after sphincteroplasty: A ten- to twenty-two-year followup study. Surgery. 1997;121(5):488-92. S0039-6060(97)90101-X [pii].

Page $7 / 11$ 
34. Tocchi A, Mazzoni G, Liotta G, Lepre L, Cassini D, Miccini M. Late development of bile duct cancer in patients who had biliary-enteric drainage for benign disease: A follow-up study of more than 1,000 patients. AnnSurg. 2001;234(2):210-4. 10.1097/00000658-200108000-00011 [doi].

35. Higuchi R, Takada T, Strasberg SM, Pitt HA, Gouma DJ, Garden OJ, et al. TG13 miscellaneous etiology of cholangitis and cholecystitis. JHepatobiliaryPancreatSci. 2013;20(1):97-105. 10.1007/s00534-012-0565-z [doi].

\section{Tables}

Table 1. Clinical and laboratory data of study subjects.

$\operatorname{BTC}(\mathrm{N}=30) \quad$ BBD $(\mathrm{N}=11) \quad$ Healthy subjects $(\mathrm{N}=10) \quad$ p value

\begin{tabular}{lllll}
\hline Age, median (min-max) & $75.5(37-87)$ & $66(49-80)$ & $63.5(58-76)$ & 0.054 \\
\hline Female, N (\%) & $10(33.3 \%)$ & $3(9.1 \%)$ & $7(70 \%)$ & 0.093 \\
\hline BMI, median (min-max) & $21.85(14.2-33.9)$ & $25.3(19.2-32.9)$ & $21.92(18.4-28.1)$ & 0.061 \\
\hline & & & & \\
\hline Tumor location, N (\%) & & & & \\
\hline Intra-hepatic & $12(40 \%)$ & & & \\
\hline Extra-hepatic & $12(40 \%)$ & & & \\
\hline Gallbladder & $6(20 \%)$ &
\end{tabular}

\begin{tabular}{ll}
\hline Stage, $N(\%)$ & \\
\hline III and $\bigotimes$ & $16(53.3 \%)$ \\
\hline I and II & $14(46.7 \%)$ \\
\hline
\end{tabular}

\begin{tabular}{|c|c|c|c|}
\hline Leucocyte (counts/ $\mu \mathrm{L}$ ) & $7303(3100-22300)$ & $5400(3700-12200)$ & 0.375 \\
\hline Neutrophil (counts/ $\mu \mathrm{L}$ ) & $5280(1700-20100)$ & $2900(1900-8900)$ & 0.203 \\
\hline Haemoglobin $(\mathrm{g} / \mathrm{dL})$ & $12.45(6.8-17.7)$ & $14.8(12.8-18.1)$ & 0.001 \\
\hline Platelet $\left(\times 10^{4}\right.$ counts $\left./ \mu \mathrm{L}\right)$ & $21.1(22.4-54.5)$ & $22.4(16.3-43.6)$ & 0.768 \\
\hline C-reactive protein (CRP) (mg/dL) & $1.30(0.05-20.85)$ & $0.13(0.1-1.24)$ & 0.003 \\
\hline Albumin (g/dL) & $3.6(1.9-4.6)$ & $4.1(3.7-4.7)$ & 0.001 \\
\hline Lactate dehydrogenase (LDH) (IU/L) & $202(113-410)$ & $187(157-281)$ & 0.164 \\
\hline T-bil (IU/L) & $0.95(0.3-31.5)$ & $0.90(0.3-1.9)$ & 0.03 \\
\hline $\mathrm{HbA} 1 \mathrm{c} \%$ & $6.0(4.3-8.5 \mathrm{ND}=1)$ & $5.7(5.5-6.8 \mathrm{ND}=2)$ & 0.192 \\
\hline Carcinoembryonic antigen (CEA) (ng/mL) & $4.3(1.9-609 \mathrm{ND}=2)$ & $2.6(1.9-7.5 \mathrm{ND}=2)$ & 0.067 \\
\hline Carbohydrate antigen 19-9 (CA19-9) (U/mL) & $63(0-17799 \mathrm{ND}=2)$ & $16(0-56 \mathrm{ND}=2)$ & 0.365 \\
\hline
\end{tabular}

median (min-max), ND: no data

BTC: biliary tract cancer, BBD: benign biliary disease, BMI: body mass index, CRP: C-reactive protein, LDH: lactate dehydrogenase, CEA: carcinoembryonic antigen.

Table 2. OTUs assigned to Enterobacteriaceae in bile and faecal samples

Highlighting indicates the OTUs detected in both faecal and bile samples

\section{Figures}


Composition rate (\%)

\begin{tabular}{|c|c|c|c|c|c|c|c|c|c|c|c|c|c|c|}
\hline & Group & BBD & & & & & & BTC & & & & & & \\
\hline & Subject ID & 20 & & 23 & & 34 & & 29 & & 37 & & 40 & & 41 \\
\hline OUT ID & Taxon & Bile & Faeces & Bile & Faeces & Bile & Faeces & Bile & Faeces & Bile & Faeces & Bile & Faeces & Bile \\
\hline OTU_0002 & $\begin{array}{l}\text { f_Enterobacteriaceae; } \\
\text { g_Escherichia; s_coli }\end{array}$ & 45.75 & & & 0.53 & 0.56 & & 6.29 & 25.91 & & & 88.05 & 19.59 & \\
\hline OTU_0004 & f_Enterobacteriaceae & & & & & & & & & & & & & 62.: \\
\hline OTU_0006 & $\begin{array}{l}\text { f_Enterobacteriaceae; } \\
\text { g_Escherichia; s_coli }\end{array}$ & 21.19 & & & & & & 1.12 & 4.32 & & & & & \\
\hline OTU_0007 & $\begin{array}{l}\text { f_Enterobacteriaceae; } \\
\text { g__Citrobacter; s_ }\end{array}$ & & & & & & & & & 5.77 & 12.37 & & & \\
\hline OTU_0013 & f_Enterobacteriaceae & & & & & & & & & & & & & \\
\hline OTU_0014 & f_Enterobacteriaceae & & & & & & & & & & & & & \\
\hline OTU_0021 & f_Enterobacteriaceae & & & & & & & 12.01 & 0.65 & & & & & \\
\hline OTU_0027 & f_Enterobacteriaceae & & & & & & & 9.52 & 0.76 & & & & & \\
\hline OTU_0034 & $\begin{array}{l}\text { f_Enterobacteriaceae; } \\
\text { g_Escherichia; s_coli }\end{array}$ & & & & & & & & & & & 2.41 & & \\
\hline OTU_0045 & $\begin{array}{l}\text { f_Enterobacteriaceae; } \\
\text { g__Citrobacter; s_- }\end{array}$ & & & & & & & & & 0.88 & 2.26 & & & \\
\hline OTU_0048 & $\begin{array}{l}\text { f_Enterobacteriaceae; } \\
\text { g__Citrobacter; s__ }\end{array}$ & & & & & & & & & 0.83 & 1.66 & & & \\
\hline OTU_0050 & f_Enterobacteriaceae & & & & & & & 4.10 & & & & & & \\
\hline OTU_0082 & $\begin{array}{l}\text { f_Enterobacteriaceae; } \\
\text { g__Klebsiella; s_ }\end{array}$ & & & & & 1.57 & & & & & & 0.17 & 0.21 & \\
\hline OTU_0091 & f_Enterobacteriaceae & & & & & & & 1.96 & & & & & & \\
\hline OTU_0096 & $\begin{array}{l}\text { f_Enterobacteriaceae; } \\
\text { g__Klebsiella; s_ }\end{array}$ & & & & & 1.95 & & & & & & & & \\
\hline OTU_0097 & $\begin{array}{l}\text { f_Enterobacteriaceae; } \\
\text { g__Klebsiella; s_ }\end{array}$ & & & & & 1.30 & 0.61 & & & & & & & \\
\hline OTU_0131 & f_Enterobacteriaceae & & & & & & & & & 0.18 & 0.52 & & & \\
\hline OTU_0148 & f_Enterobacteriaceae & & & & & & & & & 0.16 & 0.33 & & & \\
\hline OTU_0209 & $\begin{array}{l}\text { f_Enterobacteriaceae; } \\
\text { g__Citrobacter; s_ }\end{array}$ & & & & & & 0.46 & & & & & & & \\
\hline OTU_0231 & f_Enterobacteriaceae & & & & & & & 0.37 & & & & & & \\
\hline OTU_0261 & $\begin{array}{l}\text { f_Enterobacteriaceae; } \\
\text { g__Klebsiella; s_ }\end{array}$ & & & & & & & & & & & & 0.28 & \\
\hline OTU_0323 & $\begin{array}{l}\text { f_Enterobacteriaceae; } \\
\text { g__Citrobacter; s__ }\end{array}$ & & & & & & 0.18 & & & & & & & \\
\hline OTU_0376 & $\begin{array}{l}\text { f_Enterobacteriaceae; } \\
\text { g__Serratia; s_ }\end{array}$ & & & & & & 0.13 & & & & & & & \\
\hline OTU_0405 & $\begin{array}{l}\text { f_Enterobacteriaceae; } \\
\text { g_Citrobacter; s_- }\end{array}$ & & & & & & 0.11 & & & & & & & \\
\hline
\end{tabular}



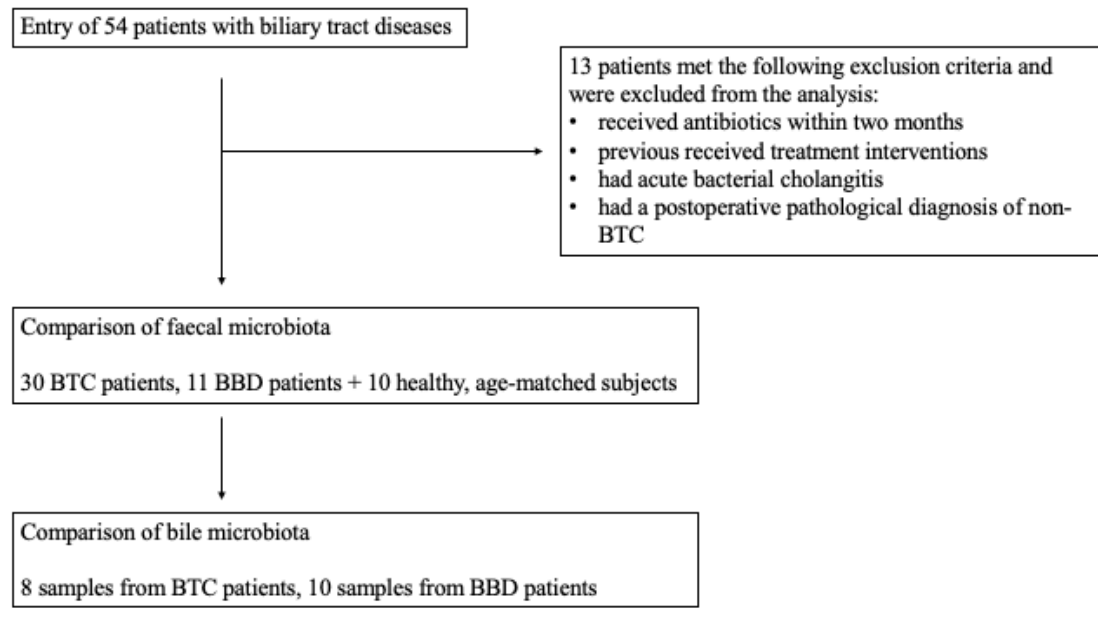

\section{Figure 1}

Overview of patient selection and analysis of each sample in this study.

(A)

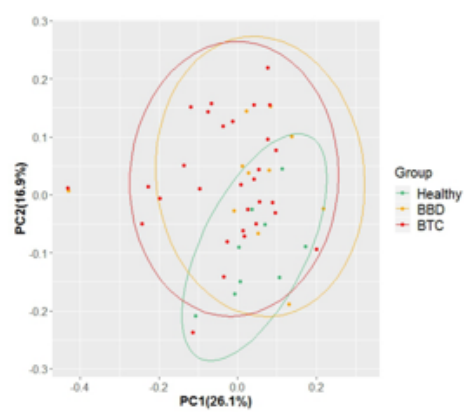

$<$ Weighted UniFrac distance > $\mathrm{p}=0.0124^{*}$
(B)

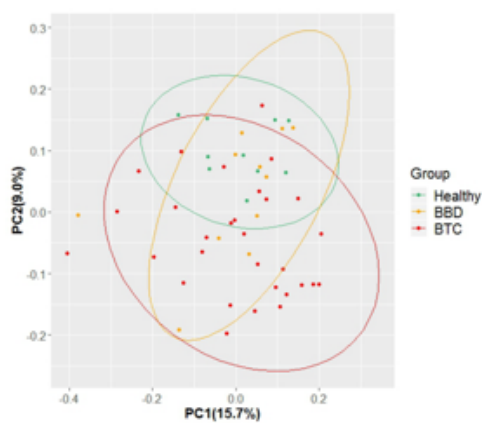

<Unweighted UniFrac distance > $\mathrm{p}=0.0293^{*}$
(C)

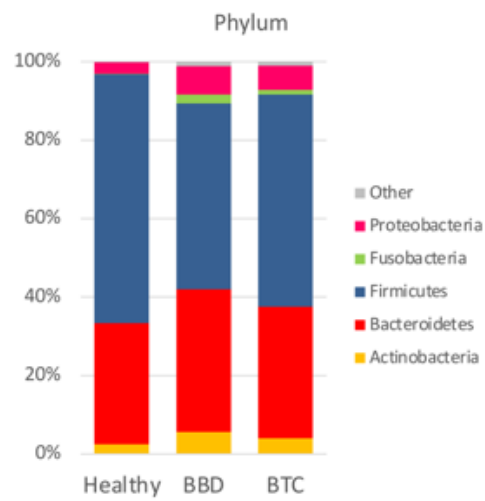

Figure 2

Overview of the faecal microbiota in BTC patients, BBD patients and healthy groups.

(A)

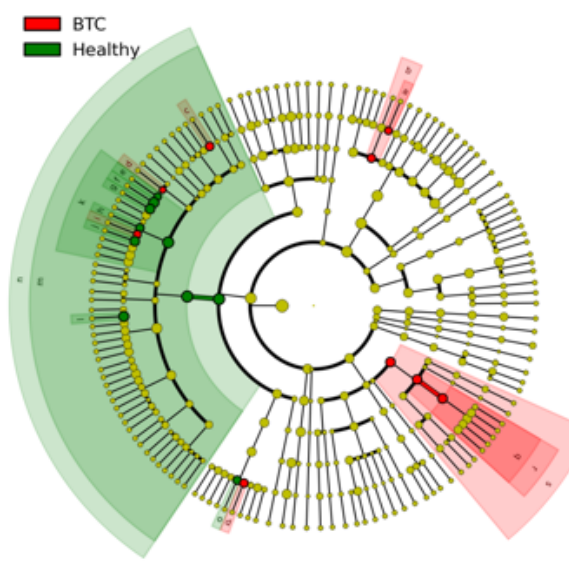

(B)

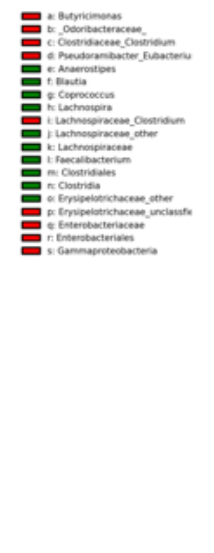

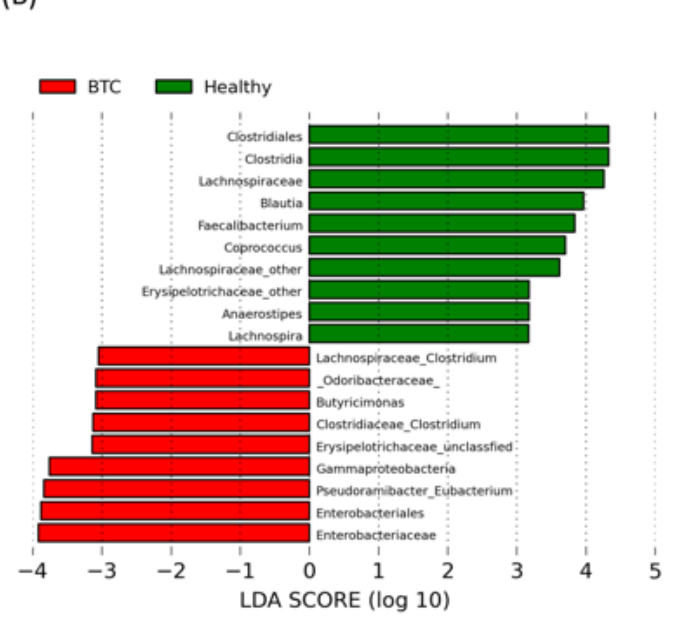


Characteristics of the faecal microbiota in each group.

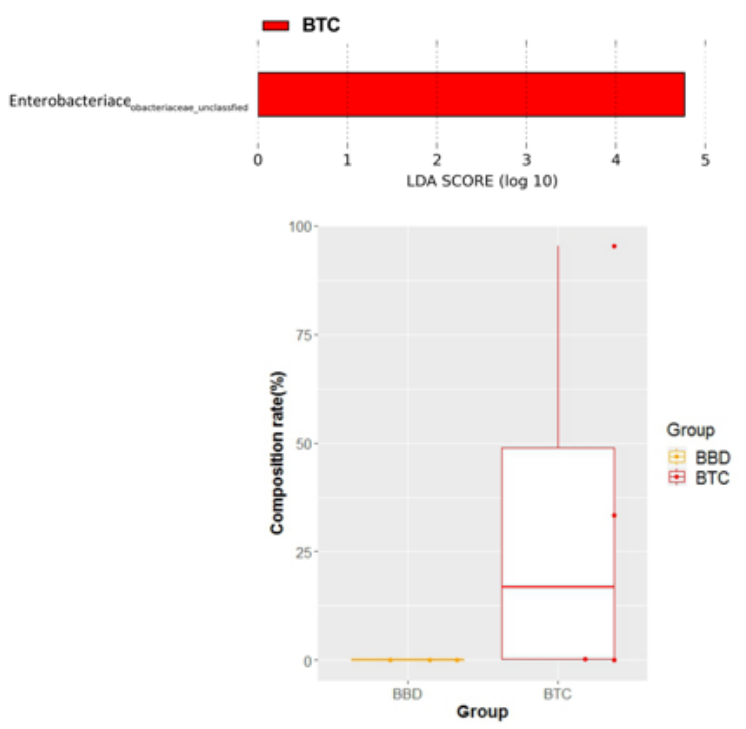

Figure 4

OTUs assigned to Enterobacteriaceae that showed a significant difference between the BBD and BTC groups in bile samples.

\section{Supplementary Files}

This is a list of supplementary files associated with this preprint. Click to download.

- OnlineSupFigure1.png

- OnlineSupFigure2.png

- OnlineSupFigure3.png

- OnlineSupFigure4.png

- SupplementaryTable1.docx 